Research Article

\title{
Electronic and Optical Properties of Sodium Niobate: A Density Functional Theory Study
}

\author{
Daniel Fritsch \\ Department of Chemistry, University of Bath, Claverton Down, Bath BA2 7AY, UK \\ Correspondence should be addressed to Daniel Fritsch; d.fritsch@bath.ac.uk
}

Received 9 November 2017; Accepted 27 December 2017; Published 7 March 2018

Academic Editor: Pavel Lejcek

Copyright (c) 2018 Daniel Fritsch. This is an open access article distributed under the Creative Commons Attribution License, which permits unrestricted use, distribution, and reproduction in any medium, provided the original work is properly cited.

In recent years, much effort has been devoted to replace the most commonly used piezoelectric ceramic lead zirconate titanate $\mathrm{Pb}\left[\mathrm{Zr}_{x} \mathrm{Ti}_{1-x}\right] \mathrm{O}_{3}(\mathrm{PZT})$ with a suitable lead-free alternative for memory or piezoelectric applications. One possible alternative to PZT is sodium niobate as it exhibits electrical and mechanical properties that make it an interesting material for technological applications. The high-temperature simple cubic perovskite structure undergoes a series of structural phase transitions with decreasing temperature. However, particularly the phases at room temperature and below are not yet fully characterised and understood. Here, we perform density functional theory calculations for the possible phases at room temperature and below and report on the structural, electronic, and optical properties of the different phases in comparison to experimental findings.

\section{Introduction}

The most widely used piezoelectric ceramic to date is lead zirconate titanate $\mathrm{Pb}\left[\mathrm{Zr}_{x} \mathrm{Ti}_{1-x}\right] \mathrm{O}_{3}$ (PZT), where the composition $x$ is used to tailor specific properties for memory or piezoelectric devices. However, due to the toxicity of these lead-containing devices, much effort has been devoted in recent years to find suitable lead-free alternatives to PZT. One promising alternative materials system is the solid solution sodium potassium niobate $(\mathrm{Na}, \mathrm{K}) \mathrm{NbO}_{3}[1,2]$. While the structural and electronic properties of the one end member potassium niobate $\left(\mathrm{KNbO}_{3}\right)$ are relatively well known, this is much less the case for the ferroelectric (FE) perovskite sodium niobate $\left(\mathrm{NaNbO}_{3}\right)$.

Like many other perovskites, $\mathrm{NaNbO}_{3}$ exhibits a large range of structural phase transitions, accompanied by changes in the ferroelectric behaviour. A first comprehensive discussion of the different structural phase transitions in $\mathrm{NaNbO}_{3}$ was reported by Megaw [3]. According to Megaw [3], the high-temperature phase of $\mathrm{NaNbO}_{3}$ is paraelectric $(\mathrm{PE})$ and crystallises in the simple cubic perovskite structure $(P m \overline{3} m)$, before it undergoes a phase transition to a PE tetragonal $T_{2}$ phase $(P 4 / \mathrm{mbm})$ at a transition temperature $T_{c 1}=913 \mathrm{~K}$. Next, there appear three distinct phase transitions into orthorhombic phases: to the PE $T_{1}$ phase $(\mathrm{Cmcm})$ at $T_{c 2}=848 \mathrm{~K}$, to the PE $S$ phase $(P n m m)$ at $T_{c 3}=793 \mathrm{~K}$, and to the antiferroelectric (AFE) $R$ phase $(P m n m)$ at $T_{c 4}=753 \mathrm{~K}$, respectively. The orthorhombic AFE $R$ phase (Pmnm) undergoes a phase transition into the orthorhombic AFE $P$ phase $(\mathrm{Pbcm})$ at $T_{c 5}=633 \mathrm{~K}$, which is the commonly assumed crystal structure at room temperature and stays stable over a wide temperature range down to $T_{c 6}=173 \mathrm{~K}$. Below $T_{c 6}$, $\mathrm{NaNbO}_{3}$ crystallises in the rhombohedral FE $N$ phase $(R 3 c)$.

However, while the structure of the high-temperature crystalline phases of $\mathrm{NaNbO}_{3}$ is commonly agreed on, there is still an ongoing discussion about the crystalline phases at room temperature and below. Darlington et al. [4] and Cheon et al. [5] reported on a possible admixture of a monoclinic phase $(\mathrm{Pm})$ into the room-temperature orthorhombic AFE $P$ phase $(P b c m)$ based on X-ray diffraction and neutron powder diffraction measurements. There are also reports about a room-temperature phase transition into a FE phase $\left(P 2_{1} m a\right)$ induced by an applied electric field $[6,7]$, by nanoparticle growth [8] or by growth as a strained thin film [9], respectively. A full list of recent experimental data can be found in $[2,10-12]$.

While there is quite a lot information available on the structural phase transitions from experiment, this is much 
less the case for theoretical investigations. Diéguez et al. [13] reported a first-principle study of epitaxial strain in perovskites, including $\mathrm{KNbO}_{3}$ and $\mathrm{NaNbO}_{3}$, while Li et al. [14] reported density functional theory (DFT) calculations for epitaxially strained $\mathrm{KNbO}_{3} / \mathrm{NaNbO}_{3}$ superlattices, thereby including the unstrained simple cubic perovskite phase $(P m \overline{3} m)$ as well. Finally, Machado et al. [15] reported on the relative phase stability and lattice dynamics of $\mathrm{NaNbO}_{3}$ from first principles. A rigorous assessment of the performance of different exchange-correlation functionals within DFT calculations and applied to the possible crystalline phases of $\mathrm{NaNbO}_{3}$ at room temperature and below is still missing to date.

The focus of the present work is on the reported possible crystalline phases of $\mathrm{NaNbO}_{3}$ at room temperature and below, especially on the coexistence of the rhombohedral FE $N$ phase $(R 3 c)$ with the monoclinic AFE $P$ phase $(P m)$ and the orthorhombic AFE $P$ phase $(P b c m)$. Since the delicate interplay of structural and electronic properties determines properties like the spontaneous polarisation, an improved description would help demystify the crystalline phases of this material at room temperature and below and to tailor it better for technical applications. Here, we present results of DFT calculations for the structural, electronic, and optical properties of the crystalline phases of $\mathrm{NaNbO}_{3}$ at room temperature and below, with a special emphasis on the performance of different flavours of the generalised gradient approximation (GGA) to the unknown exchange-correlation potential. The results include calculations based on the conventional PBE parametrisation of Perdew et al. [16], the AM05 parametrisation [17], and the PBE parametrisation revised for solids (PBEsol) [18]. In addition, we also perform benchmark calculations for the simple cubic PE perovskite phase $(P m \overline{3} m)$ utilising the hybrid functional PBE0 [19], where a quarter of the exchange potential is replaced by Hartree-Fock exact-exchange to better account for electronic correlation effects [20]. In accordance with similar investigations for the other end member $\mathrm{KNbO}_{3}$ [21] of the solid solution sodium potassium niobate $(\mathrm{Na}, \mathrm{K}) \mathrm{NbO}_{3}$, we find that the improved GGA approximations of AM05 and PBEsol perform better for the structural, electronic, and optical properties compared to the conventional PBE approximation. The results will be beneficial for future theoretical works concerning strain influences from underlying substrates or calculations of the spontaneous polarisation.

This paper is organised as follows. Section 2 introduces the necessary theoretical background and details of the calculations. Section 3 is devoted to the discussion of the obtained structural properties in comparison to available experimental data, the electronic properties, and finally, the optical properties. The final section provides a summary of the presented results and their main conclusion.

\section{Materials and Methods}

2.1. Computational Details. The results of the present work have been obtained by DFT calculations employing the Vienna ab initio simulation package (VASP 5) [22-24] together with the projector-augmented wave (PAW) formalism [25]. For the latter, standard PAW potentials supplied with VASP were used, providing 9 valence electrons for $\mathrm{Na}$ atoms $\left(2 s^{2} 2 p^{6} 3 s^{1}\right), 13$ valence electrons for $\mathrm{Nb}$ atoms $\left(4 s^{2} 4 p^{6} 5 s^{1} 4 d^{4}\right)$, and 6 valence electrons for $\mathrm{O}$ atoms $\left(2 s^{2} 2 p^{4}\right)$, respectively.

Structural relaxations have been performed within a scalar-relativistic approximation with a plane wave energy cutoff of $500 \mathrm{eV}$. $\Gamma$-centred $k$ point meshes have been used to sample the Brillouin zone and amounted to $6 \times 6 \times 6$ for the simple cubic perovskite $P m \overline{3} m$ phase, $6 \times 2 \times 6$ for the monoclinic $P m$ phase, $6 \times 6 \times 2$ for the orthorhombic Pbcm phase, and $6 \times 6 \times 2$ for the rhombohedral $R 3 c$ phase, respectively.

To evaluate the performance of different exchangecorrelation functionals, the structural and electronic properties have been calculated employing the GGA in the conventional PBE parametrisation of Perdew et al. [16], the AM05 parametrisation [17], and the $\mathrm{PBE}$ parametrisation revised for solids (PBEsol) [18]. Both AM05 and PBEsol have been developed to increase accuracy in structural properties for crystalline solids $[18,26]$. For the smallest unit cell of the simple cubic perovskite $P m \overline{3} m$ phase of $\mathrm{NaNbO}_{3}$, the results have additionally been benchmarked against hybrid functional calculations using the PBE0 functional [19] to better account for electronic correlation effects [20].

The obtained relaxed ground state structures served as a starting point for subsequent calculations of the electronic band structures and the real and imaginary parts of the dielectric functions. Thereby, the imaginary part of the dielectric tensor (in VASP) is determined by a summation over empty states using

$$
\begin{aligned}
\varepsilon_{\alpha \beta}^{(2)}(\omega)= & \frac{4 \pi^{2} e^{2}}{\Omega} \lim _{q \rightarrow 0} \frac{1}{q^{2}} \sum_{c, v, \mathbf{k}} 2 w_{\mathbf{k}} \delta\left(\epsilon_{c \mathbf{k}}-\epsilon_{v \mathbf{k}}-\omega\right) \\
& \times\left\langle u_{c \mathbf{k}+\mathbf{e}_{\alpha} q} \mid u_{v \mathbf{k}}\right\rangle\left\langle u_{c \mathbf{k}+\mathbf{e}_{\beta} q} \mid u_{v \mathbf{k}}\right\rangle^{*},
\end{aligned}
$$

where $c$ and $v$ denote the conduction and valence band states, respectively, and $u_{c \mathbf{k}}$ is the cell periodic part of the orbitals at $\mathbf{k}$. In order to ensure converged results, the number of empty bands in the calculations has been increased by a factor of three. The real part of the dielectric tensor is obtained via a Kramers-Kronig transformation:

$$
\varepsilon_{\alpha \beta}^{(1)}(\omega)=1+\frac{2}{\pi} P \int_{0}^{\infty} \frac{\varepsilon_{\alpha \beta}^{(2)}\left(\omega^{\prime}\right) \omega^{\prime}}{\omega^{\prime 2}-\omega^{2}+i \eta} d \omega^{\prime},
$$

where $P$ denotes the principal value of the integral. Details of the method can be found in [27]. The real and imaginary parts of the dielectric functions for the noncubic phases have been obtained by diagonalising the dielectric tensors for every energy point and averaging over the resulting main diagonal values, respectively.

2.2. Crystalline Phases at Room Temperature and Below. The present work focuses on the following crystalline phases at room temperature and below: the rhombohedral FE $N$ phase (R3c, SG 161, $Z=6)$, the monoclinic AFE $P$ phase $(P m$, SG $6, Z=8)$, and the orthorhombic AFE $P$ phase $(P b c m, S G$ $57, Z=8)$. In addition, benchmark calculations have been 


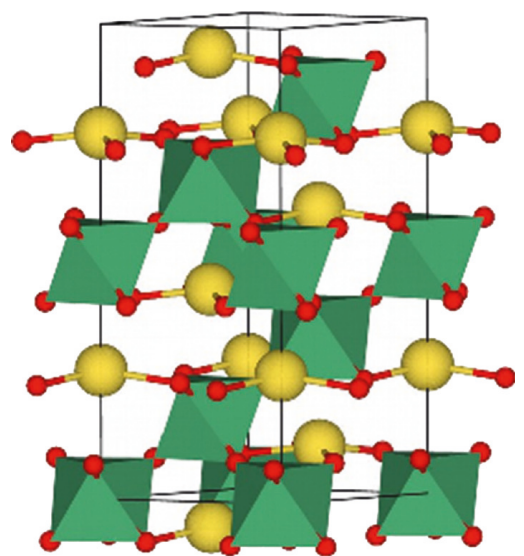

(a)

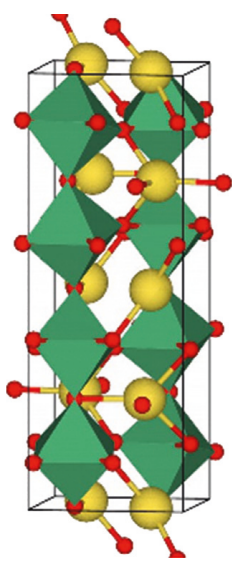

(b)

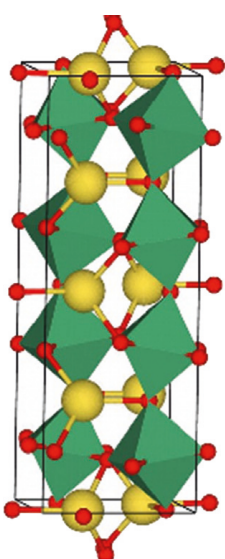

(c)

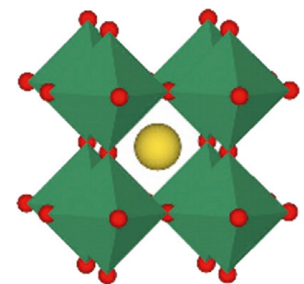

(d)

Figure 1: Crystalline phases of $\mathrm{NaNbO}_{3}$ at room temperature and below in comparison to the cubic perovskite phase ( $P m \overline{3} m$, SG 221) (d). The rhombohedral R3c phase (SG 161) (a), the orthorhombic Pbcm phase (SG 57) (b), and the monoclinic Pm phase (SG 6) (c) are shown. Pictures have been generated using VESTA [32].

carried out for the high-temperature simple cubic perovskite phase $(P m \overline{3} m$, SG 221, $Z=1)$. All four phases have been initially set up using experimental data reported by Jiang et al. [28] for the $P m \overline{3} m$ phase, by Cheon et al. [5] for the Pm phase, by Johnston et al. [6] for the $P b c m$ phase, and by Darlington and Megaw [4] for the $R 3 c$ phase, respectively. For all four phases, we performed a full geometry optimisation for several unit-cell volumes centred around the experimentally reported ones. The geometries have been fully optimised employing three different GGA functionals (PBE, PBEsol, and AM05), until the forces on each atom were smaller than $0.001 \mathrm{eV}^{-1}$. In addition, for the simple cubic perovskite $P m \overline{3} m$ phase, we also employed the PBE0 hybrid functional. Together with the plane wave energy cutoff and the $k$ point meshes reported in Section 2.1, this ensured well-converged structural and electronic properties. Exemplary, the PBEsol relaxed structures for the four different phases are shown in Figure 1, and its CIF files can be found in the Supplemental Material (available here). The volume dependence of the total energies for the four different phases gives access to the bulk modulus $B_{0}$, defined as

$$
B_{0}=V_{0}\left|\left(\frac{\partial^{2} E_{\text {tot }}}{\partial V^{2}}\right)\right|_{\left(V=V_{0}\right)},
$$

where $E_{\text {tot }}$ is the total energy and $V_{0}$ is the equilibrium bulk volume. For cubic crystals, the bulk modulus can also be expressed in terms of the elastic moduli $C_{11}$ and $C_{12}$ [29-31]:

$$
B_{0}=\frac{1}{3}\left(C_{11}+2 C_{12}\right)
$$

Typically, the total energies are fitted to the Murnaghan equation of state [33]:

$$
E(V)=E\left(V_{0}\right)+\frac{B_{0} V}{B_{0}^{\prime}}\left[\frac{\left(V_{0} / V\right)^{B_{0}^{\prime}}}{B_{0}^{\prime}-1}+1\right]-\frac{B_{0} V_{0}}{B_{0}^{\prime}-1},
$$

giving access to the bulk modulus $B_{0}$ and its pressure derivative $B_{0}^{\prime}$ as well as the ground state unit-cell volume $V_{0}$, respectively.

\section{Results and Discussion}

3.1. Simple Cubic Perovskite Pm $\overline{3} m$ Phase. The structural properties for the simple cubic perovskite $P m \overline{3} m$ phase of $\mathrm{NaNbO}_{3}$ (SG 221, $Z=1$ ) have been calculated for a range of unit-cell volumes around the initial experimental unit-cell volume [28] using different exchange-correlation functionals. The total energy curves shown in Figure 2(d) are obtained by a cubic-spline fit to the theoretical data and are rescaled to zero energy corresponding to the lowest energy for each functional.

From the total energy curves, the ground state lattice constants and bulk moduli $B_{0}$ have been obtained and are compared to available experimental data in Table 1.

It can be seen from Figure 2(d) that the PBE functional overestimates the unit-cell volume, and that both PBEsol and AM05 yield improved and nearly indistinguishable structural properties for the simple cubic perovskite $P m \overline{3} m$ phase of $\mathrm{NaNbO}_{3}$. The hybrid functional PBE0 slightly overbinds, resulting in a too short lattice constant and a too large bulk modulus, similar to other investigations on oxide semiconductors [29-31]. As has been shown recently for oxide semiconductors, this might improve with a self-consistent determination of the amount of Hartree-Fock exactexchange mixed into the hybrid functional [20]. However, as already mentioned by Machado et al. [15], it is not correct to directly compare the high-temperature measurements of the simple cubic perovskite $P m \overline{3} m$ phase with the zerotemperature DFT calculations. Their GGA calculations yielded a lattice constant $a=3.9516 \AA$ and a bulk modulus $B_{0}=193.02 \mathrm{GPa}$, in favourable agreement with our results.

Based on the slightly different ground state properties, for each of the exchange-correlation functionals, the electronic band structures have been calculated and are shown in Figure 3. The most obvious result is that all three GGA functionals yield very similar electronic band structures. This is also reflected by the indirect (direct) Kohn-Sham energy gaps (Table 1) that amount to $1.652 \mathrm{eV}(2.404 \mathrm{eV}), 1.639 \mathrm{eV}$ $(2.386 \mathrm{eV})$, and $1.642 \mathrm{eV}(2.383 \mathrm{eV})$ for the PBE, the PBEsol, 


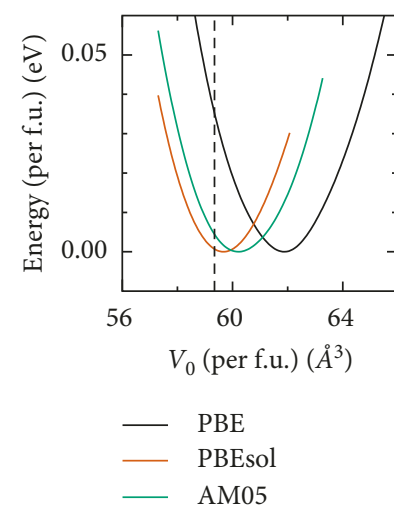

(a)

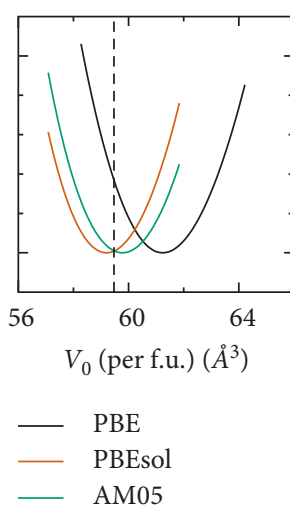

(b)

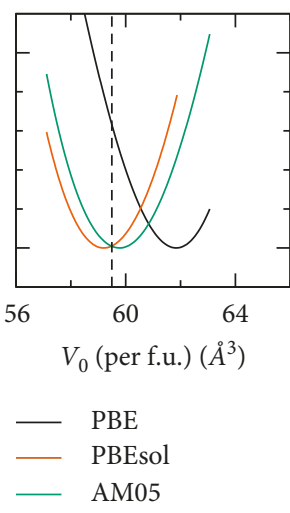

(c)

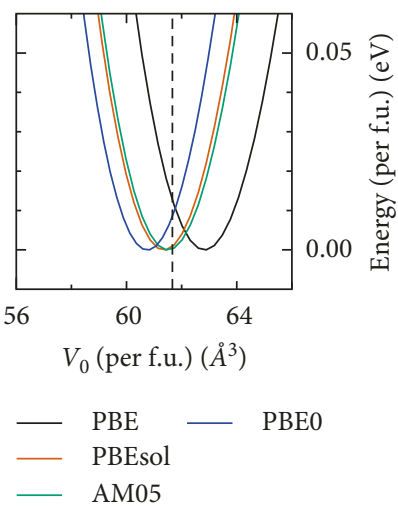

(d)

Figure 2: $\mathrm{NaNbO}_{3}$ total energy curves calculated with different exchange-correlation functionals for (a) the rhombohedral $R 3 c$ phase (SG 161), (b) the orthorhombic Pbcm phase (SG 57), (c) the monoclinic Pm phase (SG 6), and (d) the simple cubic perovskite $P m \overline{3} m$ phase (SG 221). Exchange-correlation functionals include the conventional PBE functional of Perdew et al. [16], the PBEsol functional revised for solids [18], the AM05 functional [17], and the PBE0 hybrid functional [19]. Zero energy has been rescaled corresponding to the lowest energy for each functional. The vertical black dashed lines correspond to the experimental ground state volumes.

Table 1: Structural properties of the simple cubic perovskite $P m \overline{3} m$ phase of $\mathrm{NaNbO}_{3}$ in comparison to experimental data.

\begin{tabular}{lccccc}
\hline$P m \overline{3} m$ & PBE & PBEsol & AM05 & PBE0 & Experiment \\
\hline$a(\AA)$ & 3.975 & 3.944 & 3.947 & 3.931 & $3.9507[28]$ \\
$V_{0}\left(\AA^{3}\right)$ & 62.807 & 61.349 & 61.490 & 60.745 & $61.6626[28]$ \\
$B_{0}(\mathrm{GPa})$ & 176.6 & 188.8 & 185.6 & 204.0 & $136.7^{\mathrm{a}}$ \\
& & & & 4.3 & $193.02[15], 206.3^{\mathrm{b}}$ \\
$B_{0}^{\prime}(\mathrm{GPa})$ & 4.4 & 4.6 & 4.6 & 4.567 & - \\
$E_{\mathrm{KS}}^{\text {dir }}(\mathrm{eV})$ & 2.404 & 2.386 & 2.383 & 3.756 & $3.29[34]$ \\
$E_{\mathrm{KS}}^{\text {ind }}(\mathrm{eV})$ & 1.652 & 1.639 & 1.642 & & - \\
\hline
\end{tabular}

Given are the lattice parameter $a$, equilibrium unit-cell volume $V_{0}$, the bulk modulus $B_{0}$ and its pressure derivative $B_{0}^{\prime}$, and the direct $E_{\mathrm{KS}}^{\text {dir }}(\Gamma-\Gamma)$ and indirect $E_{\mathrm{KS}}^{\text {ind }}(\mathrm{M}-\Gamma)$ Kohn-Sham energy gaps, respectively, calculated with different exchange-correlation functionals. ${ }^{\mathrm{a}}$ Calculated from experimental elastic constants $C_{11}=230 \mathrm{GPa}$ and $C_{12}=90 \mathrm{GPa}$ [11] using (4); ${ }^{\mathrm{b}}$ calculated from theoretical elastic constants $C_{11}=447 \mathrm{GPa}$ and $C_{12}=86 \mathrm{GPa}$ [13] using (4).

and the AM05 functional, respectively, and very similar valence-band bandwidths. The hybrid functional PBE0 yields a wider indirect (direct) Kohn-Sham gap of $3.756 \mathrm{eV}$ $(4.567 \mathrm{eV})$, which can also be seen in Figure 3(d), and a slightly broader valence-band bandwidth. The experimental bandgap has been measured by $\mathrm{Li}$ et al. and amounted to $3.29 \mathrm{eV}$. It is indirect as well, in agreement with our calculations. As to be expected, our GGA calculations underestimate the bandgap, while the indirect PBE0 Kohn-Sham gap of $3.756 \mathrm{eV}$ is slightly larger than the experimental value.

Based on the obtained relaxed ground state structures for the simple cubic perovskite $P m \overline{3} m$ phase, we calculated the optical properties. The real (orange) and imaginary (green) parts of the dielectric function are shown in Figure 4 for the different exchange-correlation functionals. Similarly to the electronic band structures shown in Figure 3, the three GGA functionals yield nearly indistinguishable dielectric functions. The onsets in the imaginary parts reflect the similar bandgaps already seen in the electronic band structures, and the heights and widths of the different peaks reflect the similarity in regions of nearly parallel bands in the band structures where large transition matrix elements give rise to stronger features in the imaginary part of the dielectric functions. The most striking difference in the PBE0 calculated dielectric functions is the shifted onset in the imaginary part, reflecting the larger bandgap obtained for the electronic properties. However, since the overall regions of nearly parallel bands in the band structure of Figure 3(d) are similar to the band structures of the plain GGA calculations, the peak structure in the imaginary part of the dielectric function and the widths of the peaks remain similar to the plain GGA calculations, only the heights are reduced. The PBE0-calculated dielectric functions agree best with available experimental results $[35,36]$, mostly in the onset of the imaginary part of the dielectric function (showing best agreement for the bandgap) and the low-energy onset of the real part of the dielectric function.

3.2. Monoclinic Pm Phase. The total energy curves for the monoclinic Pm phase are shown in Figure 2(c), calculated employing different GGA functionals. It can be seen that the 


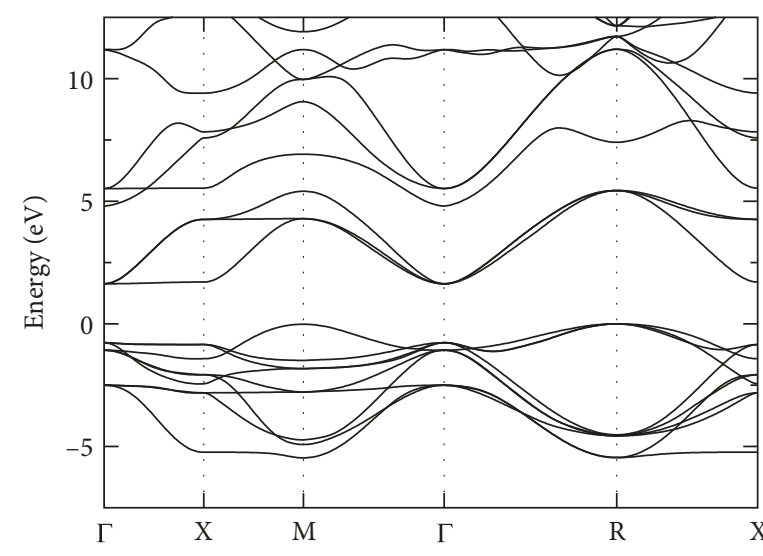

(a)

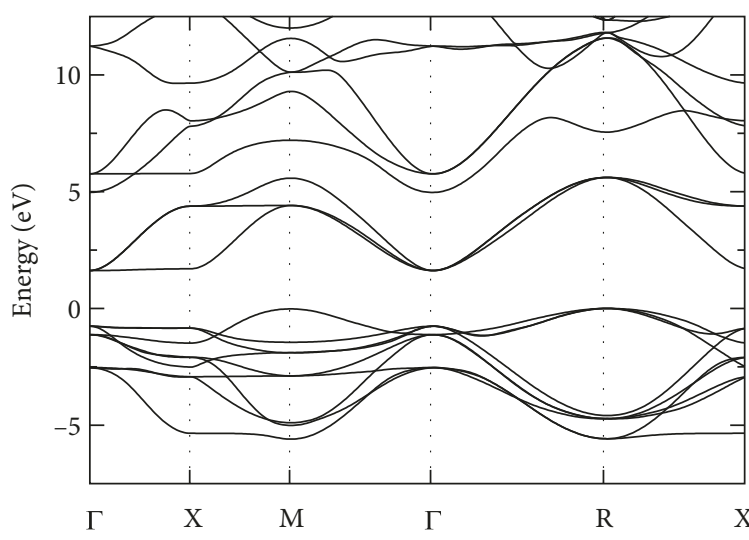

(c)

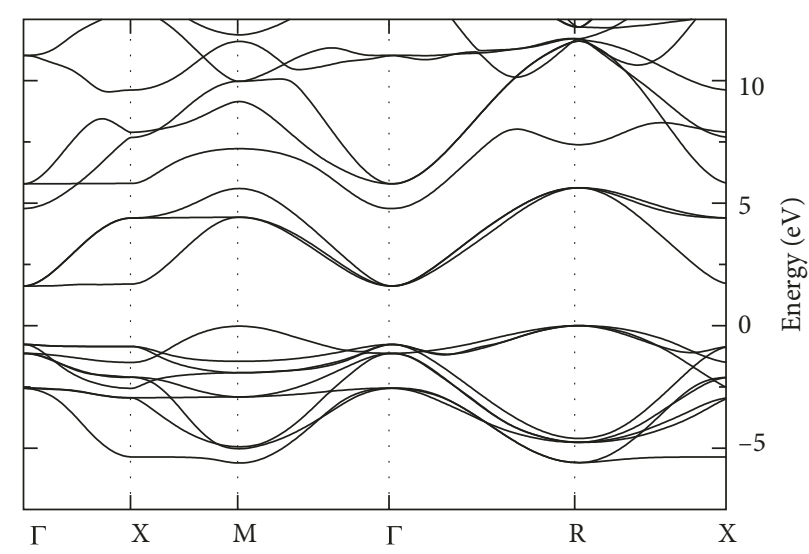

(b)

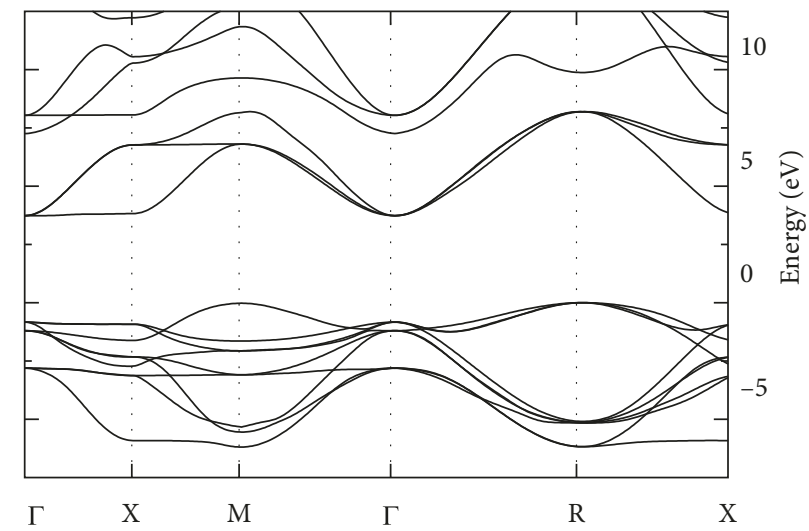

(d)

Figure 3: $\mathrm{NaNbO}_{3}$ electronic band structures calculated for the simple cubic perovskite $P m \overline{3} m$ phase employing different exchangecorrelation potentials: (a) the conventional PBE functional of Perdew et al. [16], (b) the PBEsol functional revised for solids [18], (c) the AM05 functional [17], and (d) the PBE0 hybrid functional [19]. Zero energy has been rescaled to the valence band maximum (at the M point).

difference between AM05 and PBEsol functional are larger compared to the simple cubic perovskite $P m \overline{3} m$ phase and that the conventional PBE functional again overestimates the unit-cell volume. While the PBEsol functional yields a slightly smaller ground state volume compared to the experiment, the AM05 functional ground state volume lies slightly above. The obtained ground state structural properties are given in Table 2.

The electronic band structure calculated using the PBEsol functional and based on the PBEsol ground state volume is shown in Figure 5(c). The direct Kohn-Sham energy gap of $2.290 \mathrm{eV}$ is comparable to the direct KohnSham energy gap of $2.386 \mathrm{eV}$ of the simple cubic perovskite $P m \overline{3} m$ phase, but much larger than its indirect Kohn-Sham energy gap of $1.639 \mathrm{eV}$. However, the valence-band bandwidths are very similar between the monoclinic $P m$ phase and the simple cubic perovskite $P m \overline{3} m$ phase, respectively. An additional PBE0 hybrid functional calculation based on the PBEsol ground state structure yielded an increased direct Kohn-Sham energy gap of $4.453 \mathrm{eV}$.

The real (green) and imaginary (orange) parts of the dielectric function calculated using the PBEsol functional (solid lines) are shown in Figure 6(c) for the monoclinic
Pm phase. Broad ranges of nearly parallel bands in the electronic band structure (Figure 5(c)) give rise to a very broad absorption peak centred around $5 \mathrm{eV}$. A second broad peak appears to be centred around $8.5 \mathrm{eV}$.

3.3. Orthorhombic Pbcm Phase. The total energy curves for the orthorhombic $\mathrm{Pbcm}$ phase are shown in Figure 2(b), calculated employing different GGA functionals. It can be seen that the difference between the AM05 and PBEsol functional is again larger compared to the simple cubic perovskite $P m \overline{3} m$ phase and similar to the monoclinic Pm phase. The conventional PBE functional again overestimates the unit-cell volume. Both improved approximations to the GGA functional, AM05 and PBEsol, slightly overestimate the unit-cell volume as well, with the PBEsol functional performing better. The obtained ground state structural properties are given in Table 3.

The electronic band structure calculated using the PBEsol functional and based on the PBEsol ground state structure is shown in Figure 5(b). The direct Kohn-Sham energy gap of $2.298 \mathrm{eV}$ is again comparable to the direct Kohn-Sham energy gap of $2.386 \mathrm{eV}$ of the simple cubic perovskite $P m \overline{3} m$ phase, but much larger than its indirect 


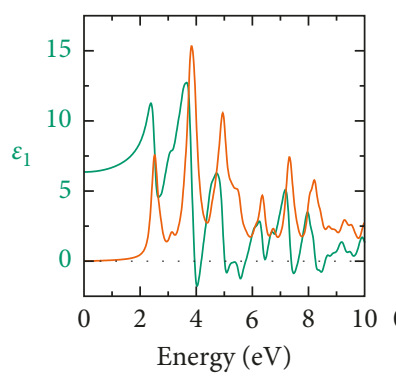

(a)

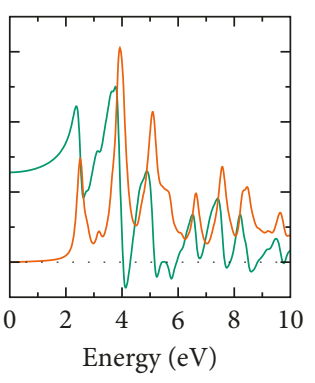

(b)

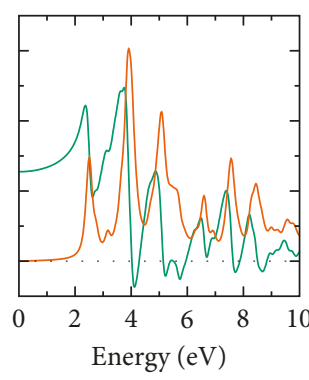

(c)

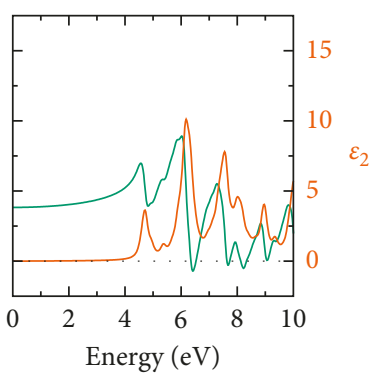

(d)

FIgURE 4: $\mathrm{NaNbO}_{3}$ dielectric functions calculated using different exchange-correlation potentials. The real (orange) and imaginary (green) parts of the dielectric functions for (a) the conventional PBE functional of Perdew et al. [16], (b) the PBEsol functional revised for solids [18], (c) the AM05 functional [17], and (d) the PBE0 hybrid functional [19].

TABle 2: Structural properties of the monoclinic $P m$ phase of $\mathrm{NaNbO}_{3}$.

\begin{tabular}{lcccc}
\hline$P m$ & PBE & PBEsol & AM05 & Experiment \\
\hline$a(\AA)$ & 5.576 & 5.495 & 5.513 & $5.50453[5]$ \\
$b(\AA)$ & 15.731 & 15.504 & 15.554 & $15.52975[5]$ \\
$c(\AA)$ & 5.640 & 5.559 & 5.576 & $5.56779[5]$ \\
$V_{0}\left(\AA^{3}\right)$ & 494.719 & 473.596 & 478.138 & $475.95682[5]$ \\
$B_{0}(\mathrm{GPa})$ & 99.8 & 117.1 & 109.4 & - \\
$B_{0}^{\prime}(\mathrm{GPa})$ & 3.3 & 6.5 & 6.4 & - \\
$E_{\mathrm{KS}}^{\text {dir }}(\mathrm{eV})$ & 2.330 & 2.290 & 2.291 & - \\
$\Delta E(\mathrm{meV})$ & -111 & -109 & -94 & - \\
\hline
\end{tabular}

Given are the lattice parameters $a, b$, and $c$, the equilibrium unit-cell volume $V_{0}$, the bulk modulus $B_{0}$ and its pressure derivative $B_{0}$, and the direct $(\Gamma-\Gamma)$ Kohn-Sham energy gaps $E_{\mathrm{KS}}^{\text {dir }}$, respectively, calculated with different exchange-correlation functionals. The last row gives the energy difference $\Delta E$ per functional unit cell with respect to the high-temperature simple cubic perovskite $P m \overline{3} m$ phase.

Kohn-Sham energy gap of $1.639 \mathrm{eV}$. However, the valenceband bandwidth is again very similar to the simple cubic perovskite $P m \overline{3} m$ phase and the monoclinic $P m$ phase, respectively. An additional PBE0 hybrid functional calculation based on the PBEsol ground state structure yielded an increased direct Kohn-Sham energy gap of $4.461 \mathrm{eV}$, very close to the PBE0 Kohn-Sham energy gap of $4.453 \mathrm{eV}$ of the monoclinic $P m$ phase. It is, however, much larger than the experimental gap of $3.45 \mathrm{eV}$, determined by Li et al. [34] using a Tauc plot and assuming an indirect bandgap.

The real (green) and imaginary (orange) parts of the dielectric function calculated using the PBEsol functional (solid lines) are shown in Figure 6(b) for the orthorhombic $\mathrm{Pbcm}$ phase. Again, similar to the monoclinic Pm phase, broad ranges of nearly parallel bands in the electronic band structure (Figure 5(b)) give rise to a very broad absorption peak centred around $5 \mathrm{eV}$. A second broad peak appears to be centred around $8.5 \mathrm{eV}$. It should be noted that the dielectric functions for the orthorhombic $P b c m$ phase and the monoclinic $P m$ phase are nearly indistinguishable. It is understandable if one assumes that the only difference in the structural properties (different orientations of the oxygen octahedra) has only little influence on the electronic and subsequently the optical properties, respectively.
3.4. Rhombohedral R3c Phase. The total energy curves for the rhombohedral $R 3 c$ phase are shown in Figure 2(a), calculated employing different GGA functionals. Similar to the orthorhombic $\mathrm{Pbcm}$ phase, the conventional PBE functional overestimates the unit-cell volume the most, whereas the AM05 and PBEsol functionals again only slightly overestimate, with the PBEsol functional performing best for the structural properties. The obtained ground state structural properties are given in Table 4.

The electronic band structure calculated using the PBEsol functional and based on the PBEsol ground state volume is shown in Figure 5(a). Due to the smaller amount of atoms in the unit cell, $Z=6$ for the rhombohedral $R 3 c$ phase compared to $Z=8$ for the orthorhombic $P b c m$ and the monoclinic $P m$ phases, there are fewer bands in the electronic band structure. The direct Kohn-Sham energy gap of $2.660 \mathrm{eV}$ is slightly larger than the direct Kohn-Sham energy gap of $2.386 \mathrm{eV}$ of the simple cubic perovskite $P m \overline{3} m$ phase and much larger than its indirect Kohn-Sham energy gap of $1.639 \mathrm{eV}$. It is also larger than the direct Kohn-Sham gaps of $2.298 \mathrm{eV}$ and $2.290 \mathrm{eV}$ for the orthorhombic $\mathrm{Pbcm}$ and the monoclinic Pm phases, respectively. The valenceband bandwidth, however, is again very similar to the simple cubic perovskite $P m \overline{3} m$, the orthorhombic $P b c m$, and the monoclinic $P m$ phases, respectively. An additional PBE0 hybrid functional calculation based on the PBEsol ground state structure yielded an increased direct Kohn-Sham energy gap of $4.840 \mathrm{eV}$, larger than the PBE0 Kohn-Sham gaps of $4.461 \mathrm{eV}$ and $4.453 \mathrm{eV}$ for the orthorhombic $\mathrm{Pbcm}$ and the monoclinic $P m$ phases, respectively.

The real (green) and imaginary (orange) parts of the dielectric function calculated using the PBEsol functional (solid lines) are shown in Figure 6(a) for the rhombohedral $R 3 c$ phase. Broad ranges of nearly parallel bands in the electronic band structure (Figure 5(a)) give rise to slightly narrower but still very broad absorption peaks compared to the orthorhombic $\mathrm{Pbcm}$ and the monoclinic Pm phases, now centred at slightly smaller energies.

\section{Conclusions}

In summary, we presented a detailed DFT investigation on the structural properties of the crystalline phases of $\mathrm{NaNbO}_{3}$ 


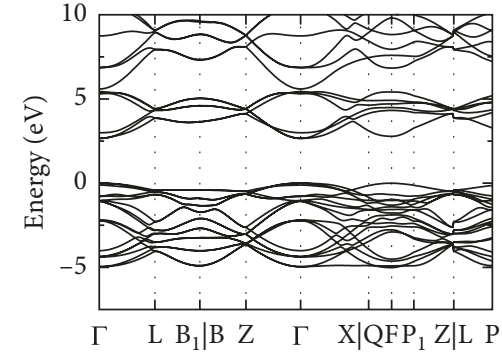

(a)

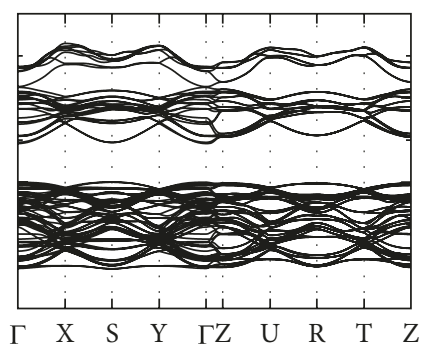

(b)

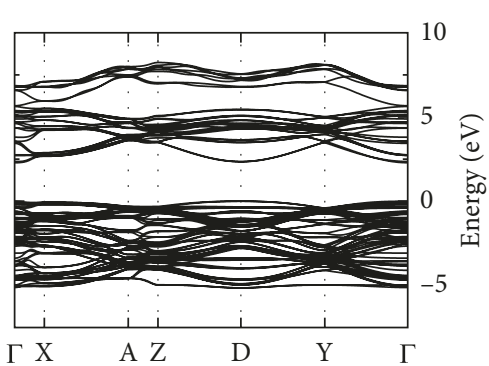

(c)

FIgURE 5: $\mathrm{NaNbO}_{3}$ electronic band structures for the crystalline phases at room temperature and below calculated using the PBEsol functional revised for solids [18]. The electronic band structures for (a) the rhombohedral $R 3 c$ phase (SG 161), (b) the orthorhombic Pbcm phase (SG 57), and (c) the monoclinic Pm phase (SG 6) are shown. Zero energy has been rescaled to the valence band maximum.

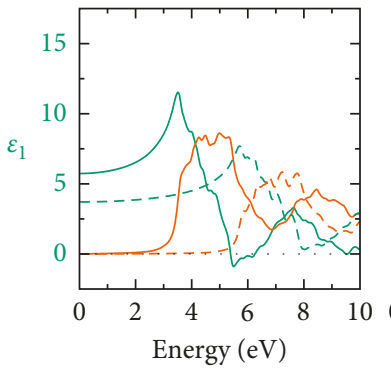

(a)

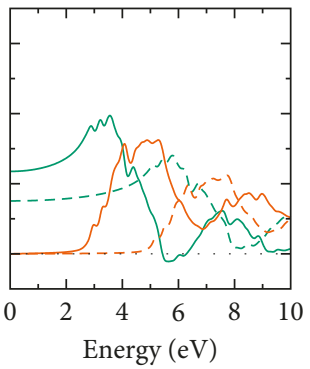

(b)

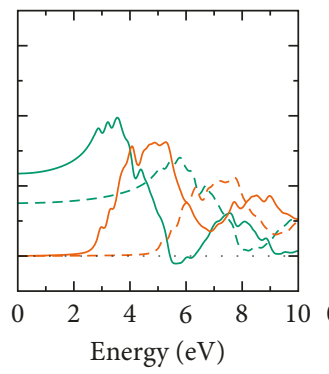

(c)

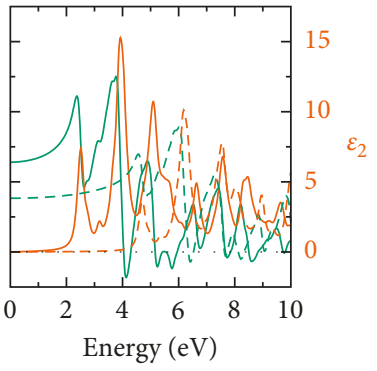

(d)

FIguRE 6: $\mathrm{NaNbO}_{3}$ dielectric functions for the crystalline phases at room temperature and below calculated using the PBEsol functional revised for solids [18] (solid lines). The real (green) and imaginary (orange) parts of the dielectric functions for (a) the rhombohedral $R 3 c$ phase (SG 161), (b) the orthorhombic Pbcm phase (SG 57), and (c) the monoclinic Pm phase (SG 6) are shown. For easier comparison, the dielectric functions of the high-temperature cubic perovskite $P m \overline{3} m$ phase (SG 221) are included in (d). In addition, PBE0 hybrid functional calculations based on the PBEsol ground state structures are shown with dashed lines.

TABLE 3: Structural properties of the orthorhombic Pbcm phase of $\mathrm{NaNbO}_{3}$.

\begin{tabular}{lcccc}
\hline$P b c m$ & PBE & PBEsol & AM05 & Experiment \\
\hline$a(\AA)$ & 5.558 & 5.496 & 5.514 & $5.505[37]$ \\
& & & & $5.506[6]$ \\
$b(\AA)$ & 5.625 & 5.562 & 5.580 & $5.569[37]$ \\
& & & & $5.566[6]$ \\
$c(\AA)$ & 15.670 & 15.494 & 15.545 & $15.519[37]$ \\
& & & & $15.520[6]$ \\
$V_{0}\left(\AA^{3}\right)$ & 489.903 & 473.632 & 478.290 & $475.771[37]$ \\
& & & & $475.632[6]$ \\
$B_{0}(\mathrm{GPa})$ & 105.1 & 117.1 & 108.9 & $157.5[38]$ \\
$B_{0}^{\prime}(\mathrm{GPa})$ & 5.4 & 6.8 & 6.9 & $4.0[38]$ \\
$E_{\mathrm{KS}}(\mathrm{eV})$ & 2.360 & 2.298 & 2.298 & $3.45[34]$ \\
$\Delta E(\mathrm{meV})$ & -108 & -109 & -94 & - \\
\hline
\end{tabular}

Given are the lattice parameters $a, b$, and $c$, the equilibrium unit-cell volume $V_{0}$, the bulk modulus $B_{0}$ and its pressure derivative $B_{0}^{\prime}$, and the direct $(\Gamma-\Gamma)$ Kohn-Sham energy gaps $E_{\mathrm{KS}}^{\mathrm{dir}}$, respectively, calculated with different exchange-correlation functionals. The last row gives the energy difference $\Delta E$ per functional unit cell with respect to the high-temperature simple cubic perovskite $\operatorname{P} m \overline{3} m$ phase.

at room temperature and below, end member in the solid solution sodium potassium niobate $(\mathrm{Na}, \mathrm{K}) \mathrm{NbO}_{3}$, and a promising lead-free alternative to PZT. The calculations
TABLE 4: Structural properties of the rhombohedral $R 3 c$ phase of $\mathrm{NaNbO}_{3}$.

\begin{tabular}{lcccc}
\hline$R 3 c$ & PBE & PBEsol & AM05 & Experiment \\
\hline$a(\AA)$ & 5.556 & 5.489 & 5.506 & $5.481[10]$ \\
$c(\AA)$ & 13.888 & 13.720 & 13.762 & $13.685[10]$ \\
$V_{0}\left(\AA^{3}\right)$ & 371.274 & 357.990 & 361.314 & $356.060[10]$ \\
$B_{0}(\mathrm{GPa})$ & 100.8 & 114.9 & 107.9 & - \\
$B_{0}^{\prime}(\mathrm{GPa})$ & 6.8 & 10.2 & 6.9 & - \\
$E_{\mathrm{KS}}^{\text {dir }}(\mathrm{eV})$ & 2.819 & 2.660 & 2.709 & - \\
$\Delta E$ & -120 & -111 & -100 & - \\
\hline
\end{tabular}

Given are the lattice parameters $a$ and $c$, the equilibrium unit-cell volume $V_{0}$, the bulk modulus $B_{0}$ and its pressure derivative $B_{0}^{\prime}$, and the direct $(\Gamma-\Gamma)$ Kohn-Sham energy gaps $E_{\mathrm{KS}}^{\mathrm{dir}}$, respectively, calculated with different exchange-correlation functionals. The last row gives the energy difference $\Delta E$ per functional unit cell with respect to the high-temperature simple cubic perovskite $P m \overline{3} m$ phase.

assessed the performance of different GGA exchangecorrelation functionals, namely, the conventional PBE parametrisation, the AM05 parametrisation, and the PBEsol parametrisation. For the simple cubic perovskite $P m \overline{3} m$ structures, additional calculations also employed the PBE0 hybrid functional. It could be shown that the improved GGA functionals AM05 and PBEsol perform better for the structural properties compared to the conventional PBE 
approximation. This is in line with similar investigations for the other end member $\mathrm{KNbO}_{3}$ [21] of the solid solution sodium potassium niobate $(\mathrm{Na}, \mathrm{K}) \mathrm{NbO}_{3}$. Based on the PBEsol ground state geometries, the electronic band structures and the optical dielectric functions have been calculated and discussed with respect to available experimental data, with the PBEsol functional performing best. This can serve as a basis for future theoretical works, calculating the spontaneous polarisations in the different phases of $\mathrm{NaNbO}_{3}$ at room temperature and below or to investigate the influence of strain effects on the structural, electronic, and optical properties, respectively.

\section{Conflicts of Interest}

The authors declare that there are no conflicts of interest regarding the publication of this paper.

\section{Acknowledgments}

This research was partly funded by the European Union's Horizon 2020 research and innovation programme under Grant Agreement no. 641864 (INREP). This work made use of the ARCHER UK National Supercomputing Service (http://www.archer.ac.uk) via the membership of the UK's HPC Materials Chemistry Consortium, funded by the Engineering and Physical Sciences Research Council (EPSRC) (EP/L000202) and the Balena HPC facility of the University of Bath.

\section{Supplementary Materials}

Electronic and optical properties of sodium niobate: a density functional theory study. Phase I: simple cubic perovskite $P m 3 m$. Phase II: rhombohedral $R 3 c$. Phase III: orthorhombic $\mathrm{Pbcm}$. Phase IV: monoclinic $\mathrm{Pm}$ phase. (Supplementary Materials)

\section{References}

[1] Y. Saito, H. Takao, T. Tani et al., "Lead-free piezoceramics," Nature, vol. 432, no. 7013, p. 84, 2004.

[2] X. Tan, C. Ma, J. Frederick, S. Beckman, and K. G. Webber, "The antiferroelectric $\leftrightarrow$ ferroelectric phase transition in leadcontaining and lead-free perovskite ceramics," Journal of the American Ceramic Society, vol. 94, no. 12, pp. 4091-4107, 2011.

[3] H. D. Megaw, "The seven phases of sodium niobate," Ferroelectrics, vol. 7, no. 1, pp. 87-89, 1974.

[4] C. N. W. Darlington and H. D. Megaw, "The low-temperature phase transition of sodium niobate and the structure of the low-temperature phase, N," Acta Crystallographica Section B Structural Crystallography and Crystal Chemistry, vol. 29, no. 10, pp. 2171-2185, 1973.

[5] C. I. Cheon, H. W. Joo, K.-W. Chae et al., "Monoclinic ferroelectric $\mathrm{NaNbO}_{3}$ at room temperature: crystal structure solved by using super high resolution neutron powder diffraction," Materials Letters, vol. 156, pp. 214-219, 2015.

[6] K. E. Johnston, C. C. Tang, J. E. Parker, K. S. Knight, P. Lightfoot, and S. E. Ashbrook, "The polar phase of $\mathrm{NaNbO}_{3}$ : a combined study by powder diffraction, solid-state NMR, and first-principles calculations," Journal of the American Chemical Society, vol. 132, no. 25, pp. 8732-8746, 2010.

[7] A. C. Sakowski-Cowley, K. Łukaszewicz, and H. D. Megaw, "The structure of sodium niobate at room temperature, and the problem of reliability in pseudosymmetric structures," Acta Crystallographica Section B Structural Crystallography and Crystal Chemistry, vol. 25, no. 5, pp. 851-865, 1969.

[8] Y. Shiratori, A. Magrez, J. Dornseiffer, F.-H. Haegel, C. Pithan, and R. Waser, "Polymorphism in micro-, submicro-, and nanocrystalline $\mathrm{NaNbO}_{3}$," Journal of Physical Chemistry B, vol. 109, no. 43, pp. 20122-20130, 2005.

[9] J. Schwarzkopf, M. Schmidbauer, T. Remmele et al., "Straininduced phase transitions in epitaxial $\mathrm{NaNbO}_{3}$ thin films grown by metal-organic chemical vapour deposition," Journal of Applied Crystallography, vol. 45, no. 5, pp. 10151023, 2012.

[10] S. K. Mishra, N. Choudhury, S. L. Chaplot, P. S. R. Krishna, and R. Mittal, "Competing antiferroelectric and ferroelectric interactions in $\mathrm{NaNbO}_{3}$ : neutron diffraction and theoretical studies," Physical Review B, vol. 76, no. 2, p. 024110, 2007.

[11] I. Tomeno, Y. Tsunoda, K. Oka, M. Matsuura, and M. Nishi, "Lattice dynamics of cubic $\mathrm{NaNbO}_{3}$ : An inelastic neutron scattering study," Physical Review B, vol. 80, no. 10, p. 104101, 2009.

[12] Y. I. Yuzyuk, P. Simon, E. Gagarina et al., "Modulated phases in $\mathrm{NaNbO}_{3}$ : Raman scattering, synchrotron X-ray diffraction, and dielectric investigations," Journal of Physics: Condensed Matter, vol. 17, no. 33, pp. 4977-4990, 2005.

[13] O. Diéguez, K. M. Rabe, and D. Vanderbilt, "First-principles study of epitaxial strain in perovskites," Physical Review B, vol. 72, no. 14, p. 144101, 2005.

[14] Z. Li, T. Lü, and W. Cao, "Phase transition and ferroelectric properties of epitaxially strained $\mathrm{KNbO}_{3} / \mathrm{NaNbO}_{3}$ superlattice," Journal of Applied Physics, vol. 104, no. 12, p. 126106, 2008.

[15] R. Machado, M. Sepliarsky, and M. G. Stachiotti, "Relative phase stability and lattice dynamics of $\mathrm{NaNbO}_{3}$ from firstprinciples calculations," Physical Review B, vol. 84, no. 13, p. 134107, 2011.

[16] J. P. Perdew, K. Burke, and M. Ernzerhof, "Generalized gradient approximation made simple," Physical Review Letters, vol. 77, no. 18, pp. 3865-3868, 1996.

[17] R. Armiento and A. E. Mattsson, "Functional designed to include surface effects in self-consistent density functional theory," Physical Review B, vol. 72, no. 8, p. 085108, 2005.

[18] J. P. Perdew, A. Ruzsinszky, G. I. Csonka et al., "Restoring the density-gradient expansion for exchange in solids and surfaces," Physical Review Letters, vol. 100, no. 13, p. 136406, 2008.

[19] C. Adamo and V. Barone, "Toward reliable density functional methods without adjustable parameters: The PBE0 model," Journal of Chemical Physics, vol. 110, no. 13, pp. 6158-6170, 1999.

[20] D. Fritsch, B. J. Morgan, and A. Walsh, "Self-consistent hybrid functional calculations: implications for structural, electronic, and optical properties of oxide semiconductors," Nanoscale Research Letters, vol. 12, no. 1, p. 19, 2017.

[21] F. Schmidt, M. Landmann, E. Rauls et al., "Consistent atomic geometries and electronic structure of five phases of potassium niobate from density-functional theory," Advances in Materials Science and Engineering, vol. 2017, Article ID 3981317, 13 pages, 2017.

[22] G. Kresse and J. Hafner, "Ab initio molecular dynamics for liquid metals,” Physical Review B, vol. 47, no. 1, pp. 558-561, 1993. 
[23] G. Kresse and J. Hafner, "Ab initio molecular-dynamics simulation of the liquid-metal-amorphous-semiconductor transition in germanium," Physical Review B, vol. 49, no. 20, pp. 14251-14269, 1994.

[24] G. Kresse and J. Furthmüller, "Efficiency of ab-initio total energy calculations for metals and semiconductors using a plane-wave basis set," Computational Materials Science, vol. 6, no. 1, pp. 15-50, 1996.

[25] P. E. Blöchl, "Projector augmented-wave method," Physical Review B, vol. 50, no. 24, pp. 17953-17959, 1994.

[26] A. E. Mattsson, R. Armiento, J. Paier, G. Kresse, J. M. Wills, and T. R. Mattsson, "The AM05 density functional applied to solids," Journal of Chemical Physics, vol. 128, no. 8, p. 084714, 2008.

[27] M. Gajdoš, K. Hummer, G. Kresse, J. Furthmüller, and F. Bechstedt, "Linear optical properties in the projectoraugmented wave methodology," Physical Review B, vol. 73, no. 4, p. 045112, 2006.

[28] L. Jiang, D. C. Mitchell, W. Dmowski, and T. Egami, "Local structure of $\mathrm{NaNbO}_{3}$ : a neutron scattering study," Physical Review B, vol. 88, no. 1, p. 014105, 2013.

[29] D. Fritsch and C. Ederer, "Epitaxial strain effects in the spinel ferrites $\mathrm{CoFe}_{2} \mathrm{O}_{4}$ and $\mathrm{NiFe}_{2} \mathrm{O}_{4}$ from first principles," Physical Review B, vol. 82, no. 10, p. 104117, 2010.

[30] D. Fritsch and C. Ederer, "Strain effects in spinel ferrite thin films from first principles calculations," Journal of Physics: Conference Series, vol. 292, p. 012014, 2011.

[31] D. Fritsch and C. Ederer, "Dielectric response of epitaxially strained $\mathrm{CoFe}_{2} \mathrm{O}_{4}$ spinel thin films," Physical Review B, vol. 86, no. 12, p. 014406, 2012.

[32] K. Momma and F. Izumi, "VESTA: a three-dimensional visualization system for electronic and structural analysis," Journal of Applied Crystallography, vol. 41, no. 3, pp. 653-658, 2008.

[33] F. D. Murnaghan, "The compressibility of media under extreme pressures," Proceedings of the National Academy of Sciences, vol. 30, no. 9, pp. 244-247, 1944.

[34] P. Li, S. Ouyang, G. Xi, T. Kako, and J. Ye, "The Effects of crystal structure and electronic structure on photocatalytic $\mathrm{H}_{2}$ evolution and $\mathrm{CO}_{2}$ reduction over two phases of perovskitestructured $\mathrm{NaNbO}_{3}$," Journal of Physical Chemistry C, vol. 116, no. 14, pp. 7621-7628, 2012.

[35] M. Tyunina, D. Chvostova, L. D. Yao et al., "Interband transitions in epitaxial ferroelectric films of $\mathrm{NaNbO}_{3}$," Physical Review B, vol. 92, no. 10, p. 104101, 2015.

[36] T. Kocourek, S. Inkinen, O. Pacherova et al., "Effects of doping and epitaxy on optical behavior of $\mathrm{NaNbO}_{3}$ films," Applied Physics Letters, vol. 107, no. 17, p. 172906, 2015.

[37] I. Lefkowitz, K. Łukaszewicz, and H. D. Megaw, "The high-temperature phases of sodium niobate and the nature of transitions in pseudosymmetric structures," Acta Crystallographica, vol. 20, no. 5, pp. 670-683, 1966.

[38] S. K. Mishra, M. K. Gupta, R. Mittal, S. L. Chaplot, and T. Hansen, "Suppression of antiferroelectric state in $\mathrm{NaNbO}_{3}$ at high pressure from in situ neutron diffraction," Applied Physics Letters, vol. 101, no. 24, p. 242907, 2012. 


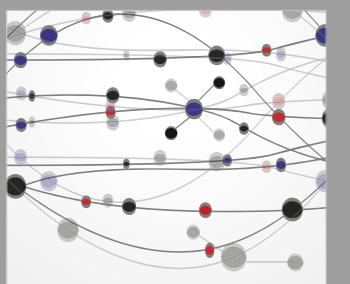

The Scientific World Journal
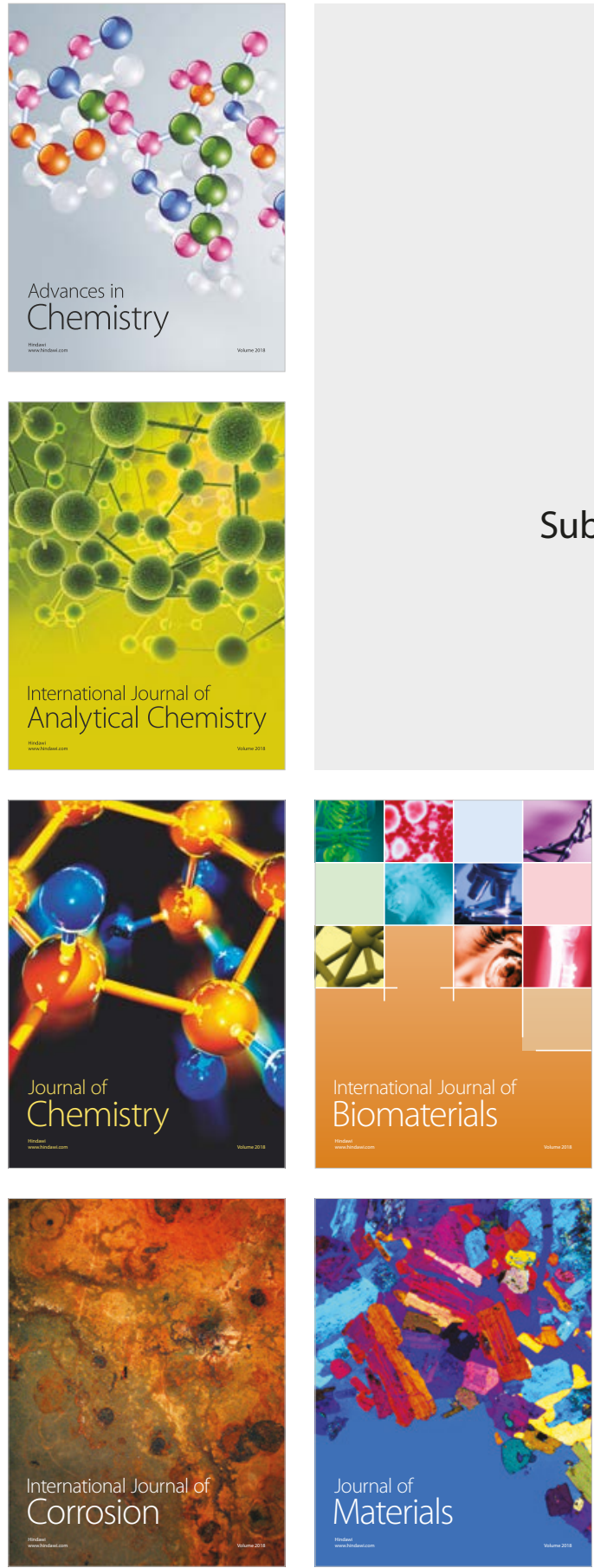

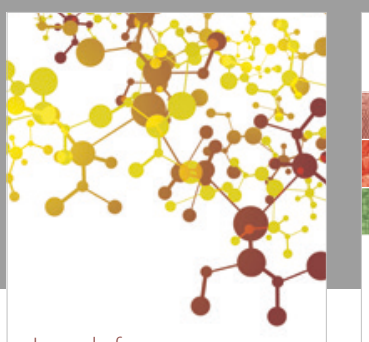

Journal of

Applied Chemistry
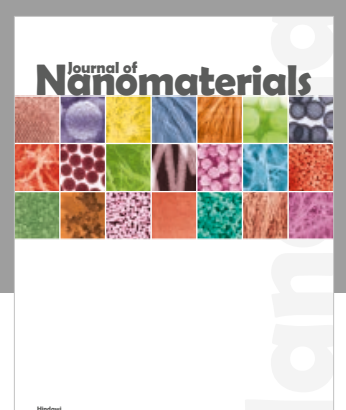

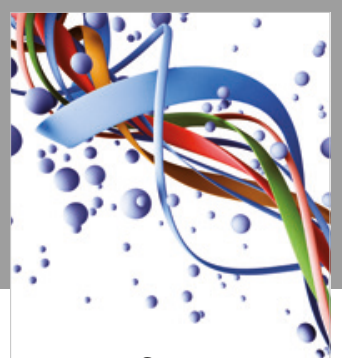

Scientifica

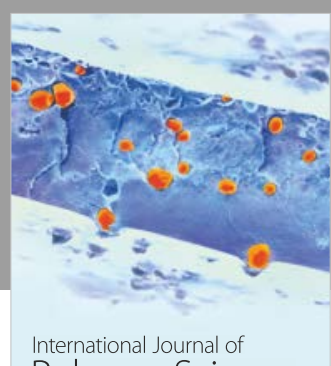

Polymer Science

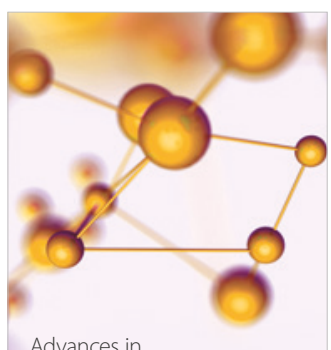

Physical Chemistry
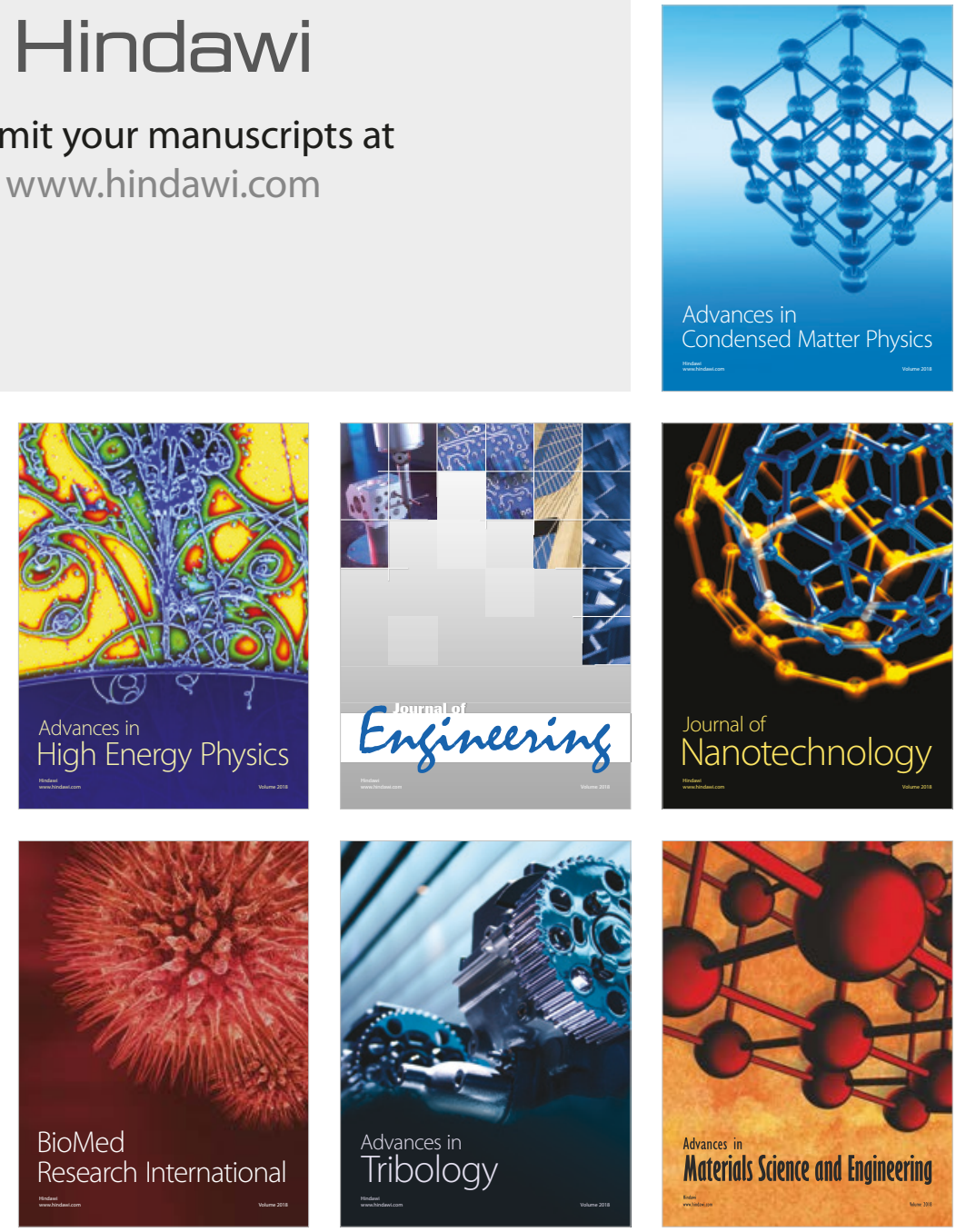